\title{
Aggregation-Enhanced Emission of Gold Nanoclusters Induced by Serum Albumin and Its Application to Protein Detection and Fabrication of Molecular Logic Gates
}

\author{
Zhenzhen Huang, Min Wang, Zilong Guo, Haonan Wang, He Dong, and Wensheng Yang*(-) \\ Key Laboratory of Surface and Interface Chemistry of Jilin Province, College of Chemistry, Jilin University, Changchun 130012, P. \\ R. China
}

\section{Supporting Information}

\begin{abstract}
Exploring aggregation-enhanced emission (AEE) of gold nanoclusters (Au NCs) is beneficial for extending their applications in sensing and molecular information processing. Herein, we present the first report of a protein-induced AEE effect of Au NCs. When human serum albumin (HSA) is mixed with glutathione-capped $\mathrm{Au}$ NCs under appropriate $\mathrm{pH}$ conditions, the $\mathrm{Au}$ NCs undergo extensive aggregation and exhibit significantly enhanced emission, attributed to the electrostatic and hydrophobic interactions between HSA and the NCs. Such an AEE effect is specific to serum albumin over a variety of other proteins, which facilitates the development of a facile approach for HSA determination. This sensing method displays satisfactory recoveries of $96.0-98.7 \%$ when it is applied to HSA detection in artificial urine. Moreover, the AEE effect is suited to the fabrication of AND and INHIBIT logic gates by using HSA and pH/ protein-binding drug as inputs and the emission as output.
\end{abstract}

\section{INTRODUCTION}

Ligand-capped luminescent gold nanoclusters (Au NCs) have attracted extensive attention for facile syntheses, high photostability, and good biocompatibility. Particularly, the luminescence of $\mathrm{Au} \mathrm{NCs}$ can readily be modified using various analytes, including metal cations, inorganic anions, and biomolecules, which provide a basis for developing sensing methods for chemical/biological detection. ${ }^{1-6}$ In most of the cases, interactions between the $\mathrm{Au} \mathrm{NCs}$ and analytes cause luminescence quenching of the NCs. For example, several $\mathrm{Hg}^{2+}$-ion-sensing methods have been designed by using the effective luminescence quenching of $\mathrm{Au}$ NCs through metallophilic $\mathrm{Hg}^{2+}-\mathrm{Au}^{+}$interactions. ${ }^{9-9}$ Chemical oxidation also causes the luminescence quenching of Au NCs, which can be used for sensing $\mathrm{CN}^{-}$and $\mathrm{H}_{2} \mathrm{O}_{2} \cdot{ }^{10,11}$ Recently, a limited but increasing number of studies have focused on the luminescence enhancement of Au NCs for sensing application. For example, Banerjee et al. report an $\mathrm{As}^{3+}$ ion assay based on the luminescence enhancement of dicysteine-capped $\mathrm{Au}$ NCs through the formation of a charge-transfer complex. ${ }^{12}$ Liu et al. develop a strategy for cysteine determination based on the enhanced emission of protein-capped Au NCs induced by attachment of cysteine on the NC surfaces. ${ }^{13}$

Aggregation-enhanced emission (AEE) is an intriguing optical phenomenon in which weakly luminescent species display remarkably enhanced emission in their aggregated states. $^{14,15}$ While AEE is usually observed for organic molecules, it was demonstrated very recently that Au NCs also exhibit the AEE effect. For example, Pei et al. reported the AEE of $\mathrm{Au}$ NCs induced by $\mathrm{Pb}^{2+}$ ions, ${ }^{16}$ while Zhou et al. reported a similar phenomenon induced by $\mathrm{Ag}^{+}$ions. ${ }^{17}$ YahiaAmmar et al. demonstrated the AEE effect of Au NCs resulting from electrostatic interactions with cationic polymers, such as poly(allylamine hydrochloride) and polyethylenimine. ${ }^{18}$

Human serum albumin (HSA) is a vital protein for human beings since it regulates intravascular osmotic pressure and transports bioactive molecules such as vitamins, hormones, and drugs in bloodstream. For a healthy person, HSA is normally present in blood rather than in urine due to filtering by the kidneys. However, when the kidneys are damaged, high levels of HSA are presented in urine, which is referred to as "albuminuria" and is a key clinical indicator of diabetes, renal, and cardiovascular diseases. ${ }^{19}$ Herein, we reported the first study using protein, that is, HSA, to induce the aggregation of glutathione-capped $\mathrm{Au} \mathrm{NCs}$ (GSH-Au NCs), which, in turn, significantly enhanced the emission of the NCs. By taking advantage of the AEE effect, we developed a luminescence enhancement strategy for protein detection, which showed excellent sensitivity and selectivity for serum albumin over a variety of other proteins. Furthermore, molecular logic gates

Received: August 3, 2018

Accepted: September 11, 2018

Published: October 8, 2018 

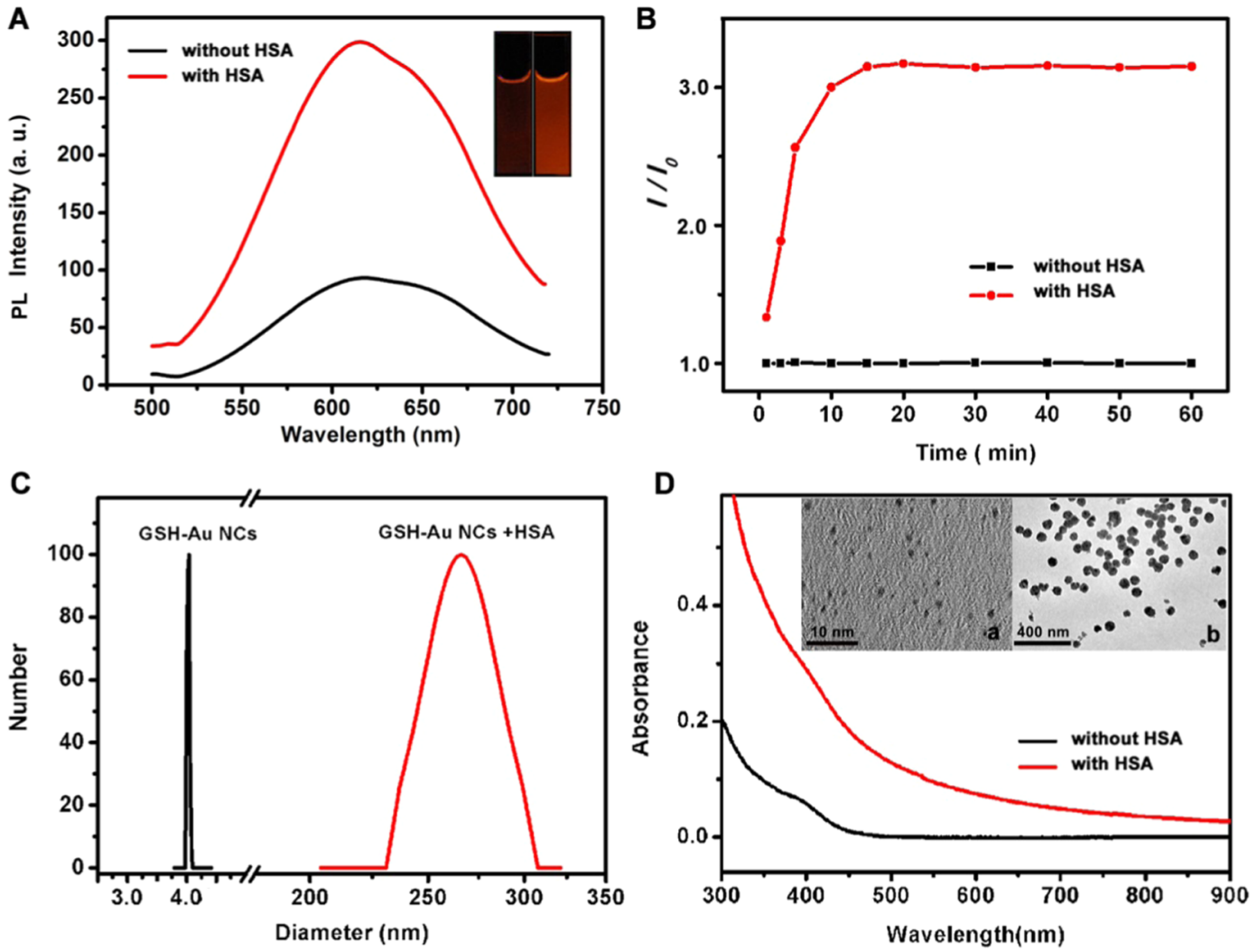

Figure 1. (A) Emission spectra of GSH-Au NC dispersion with and without HSA ( $\lambda_{\mathrm{ex}}=365 \mathrm{~nm}$ ). Inset gives optical images of the Au NC without (left) and with HSA (right) under a $365 \mathrm{~nm}$ UV lamp. (B) Temporal evolution of $I / I_{0}$ of the Au NC dispersion at $618 \mathrm{~nm}$ with and without HSA. (C) Hydrodynamic diameter profiles of the Au NCs with and without HSA. (D) UV-vis spectra of the Au NC dispersion with and without HSA. Inset gives the corresponding transmission electron microscopy (TEM) micrographs of the NC without (a) and with (b) HSA. HSA concentration was set to be $500 \mathrm{nM}$ in all the experiments.
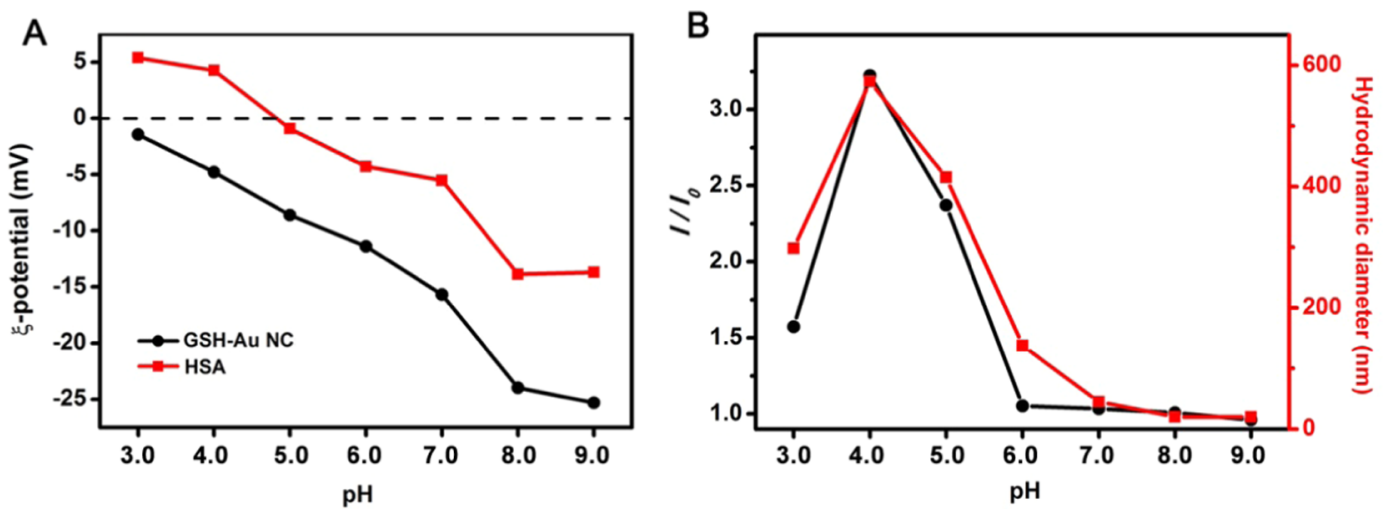

Figure 2. (A) $\zeta$-Potential of GSH-Au NC and HSA under different $\mathrm{pH}$ conditions. (B) Variations of the enhancement factor, $I / I_{0}$, and hydrodynamic diameters of the NCs in the presence of HSA, with pH. HSA concentration was set to be $500 \mathrm{nM}$ in all the experiments.

capable of performing AND and INHIBIT operations were fabricated based on the AEE effect.

\section{RESULTS AND DISCUSSION}

2.1. Aggregation-Enhanced Emission of GSH-Au NCs in the Presence of HSA. GSH-Au NCs were prepared following Xie's method. ${ }^{20}$ The as-prepared Au NCs exhibited absorption/emission bands around 390/618 nm, respectively (Figure S1). A dispersion of GSH-Au NCs in phosphate buffer ( $\mathrm{pH}$ 4.0) exhibited weak luminescence while being irradiated by ultraviolet (UV) light, which was significantly enhanced upon the addition of HSA (Figure 1A). The luminescence enhancement of the NCs by HSA was then studied by monitoring the dependence of the enhancement factor, $I / I_{0}$, with incubation time, where $I$ and $I_{0}$ represent luminescent emission intensities of the NCs at $618 \mathrm{~nm}$ with and without HSA, respectively. Figure $1 \mathrm{~B}$ reveals that the $I / I_{0}$ value increased rapidly and plateaued over $15 \mathrm{~min}$ following the addition of HSA, while the luminescence of the Au NC dispersion remained unchanged in the absence of HSA, which highlighted the vital role played by HSA in enhancing the emission of the NCs.

Dynamic light scattering (DLS) analysis indicates that the hydrodynamic diameter of the GSH-Au NC was ca. $4 \mathrm{~nm}$, 
A

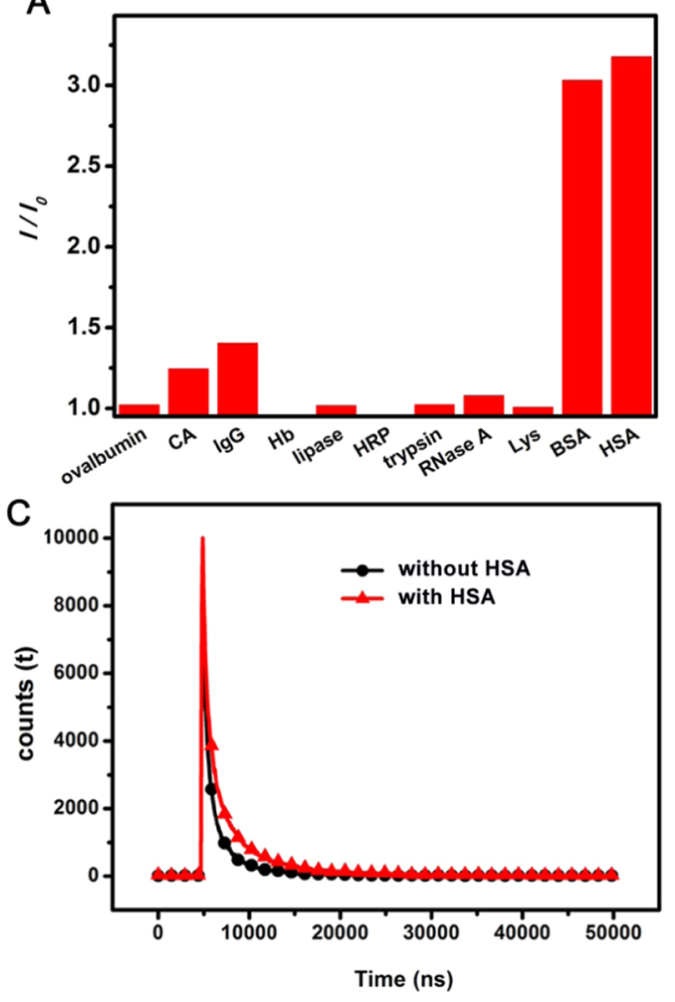

B
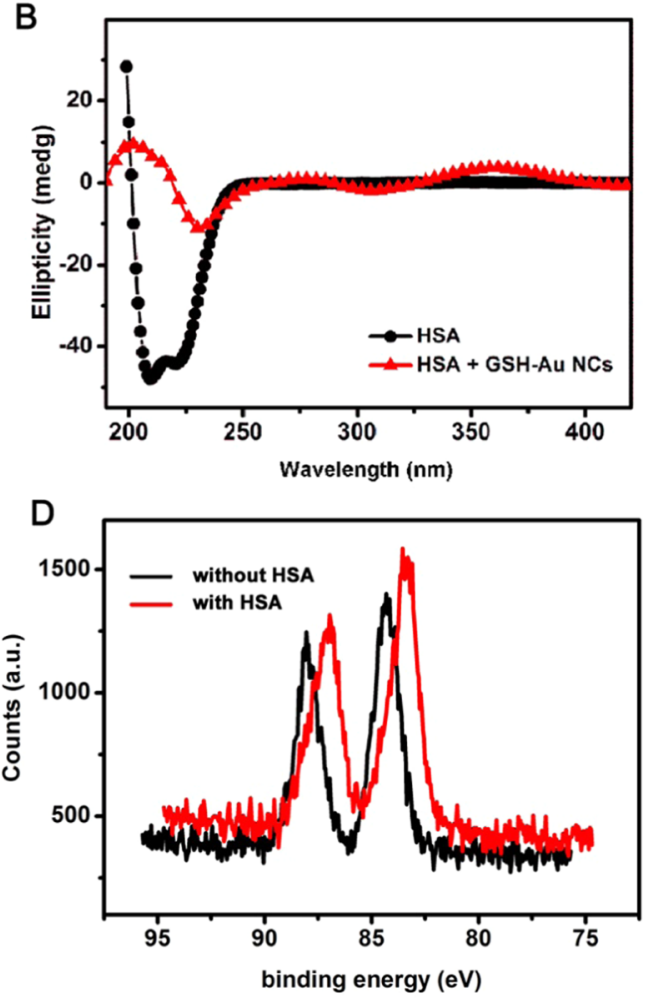

Figure 3. (A) Histogram of enhancement factor, $I / I_{0}$, at $618 \mathrm{~nm}$ of the Au NC dispersion upon addition of ovalbumin, CA, IgG, Hb, lipase, $\mathrm{HRP}$, trypsin, RNase A, Lys, BSA, and HSA. (B) Circular dichroism (CD) spectra of HSA with and without the Au NCs. (C) Luminescence decay and (D) X-ray photoelectron spectroscopy (XPS) profiles of the Au NCs with and without HSA. Concentrations of all the proteins were set to be 500 $\mathrm{nM}$ in these experiments.

which increased significantly to ca. $570 \mathrm{~nm}$ upon the addition of HSA (Figure 1C), implying that the Au NCs may undergo extensive aggregation in the presence of HSA. TEM study reveals the NCs were well dispersed and $\sim 1.1 \mathrm{~nm}$ in size, while large aggregates with sizes of $\sim 60.9 \mathrm{~nm}$ were obtained upon the addition of HSA (inset, Figure 1D). The larger hydrodynamic diameters derived from DLS measurements than those determined by TEM observations are related to the presence of hydration shells around the Au NCs and the aggregates in aqueous media. Moreover, the UV-vis absorption spectra displayed in Figure 1D reveal a noticeable increase in background scattering in the absorbance of the $\mathrm{Au}$ NC dispersion after the introduction of HSA. Correspondingly, the transparent dispersion of GSH-Au NCs became slightly turbid after the addition of HSA (Figure S2) attributed to the aggregation of the NCs, which accounted for the increased background scattering in the UV-vis absorption spectrum. Moreover, the characteristic surface plasmon resonance peak of gold nanoparticles $\sim 520 \mathrm{~nm}$ did not appear in the UV-vis absorption spectrum, which excluded the formation of large gold nanoparticles upon mixing the GSH$\mathrm{Au}$ NCs with HSA. These results were consistent with the AEE of Au NCs induced by polymers and metal ions. ${ }^{16-18}$

2.2. Possible Mechanism for the HSA-Induced AEE of GSH-Au NCs. Electrostatic interactions are essential for driving the polymer/metal cation-induced aggregation of metal nanoclusters. ${ }^{17,18}$ In our case, $\zeta$-potential measurements reveal that GSH-Au NCs were negatively charged at $\mathrm{pH}$ 3.0-9.0, while HSA is an acidic protein with an isoelectric point $(\mathrm{pI})$ of 4.7 (Figure 2A). Figure $2 \mathrm{~B}$ shows that both $I / I_{0}$ and the hydrodynamic diameters of the Au NCs following the addition of HSA were highly $\mathrm{pH}$ dependent. A maximum $I / I_{0}$ value of 3.3 was observed at $\mathrm{pH} 4.0$, in which electrostatic attractions between the negatively charged $\mathrm{Au} \mathrm{NCs}(-4.8 \mathrm{mV})$ and the positively charged HSA $(4.3 \mathrm{mV})$ lead to the aggregation of the NCs, followed by the enhanced luminescence. When $\mathrm{pH}$ of the dispersion was set to be 3.0, at which the Au NCs were less charged, or to 5.0, at which the HSA was less charged, obvious decreases in $I / I_{0}$ were observed, owing to the limited electrostatic attraction. The AEE effect was negligible at $\mathrm{pH}$ value higher than 6.0 since both the NCs and HSA were negatively charged at the high $\mathrm{pH}$. Hence, we deduced that electrostatic interactions between the GSH-Au NCs and the HSA played important roles in inducing the AEE effect.

The effect of other protein with $\mathrm{pI}$ similar to or higher than that of HSA, including ovalbumin (4.7), carbonic anhydrase (CA, 6.1), immunoglobulin G (IgG, 6.6), hemoglobin ( $\mathrm{Hb}$, 6.8), lipase (6.9), horseradish peroxidase (HRP, 7.2), trypsin (8.7), RNase A (9.2), lysozyme (Lys, 10.8) or bovine serum albumin (BSA, 4.7), on the enhancement factor was further evaluated to understand the AEE effect. After being added into aqueous dispersions of the GSH-Au NCs at $\mathrm{pH}$ 4.0, as shown in Figure 3A, only HSA and BSA, a highly structural and functional analogue of HSA, can lead to the significant emission enhancement of GSH-Au NCs, while other proteins only induced slight changes in the enhancement factor. The fact that other proteins, except serum albumin, failed to trigger the AEE of GSH-Au NC indicated that electrostatic interactions between protein and the NCs alone are insufficient to promote the AEE effect. In addition to electrostatic attraction, it is likely that there exist other driving forces to 
contribute to the AEE effect, which appear to be specific for serum albumin.

Serum albumin is a typical protein with several hydrophobic pockets and can bind to many organic probes through hydrophobic interactions. ${ }^{21-27}$ Circular dichroism (CD) spectra measurement was performed to further investigate the interaction of HSA with the Au NCs. As shown in Figure 3B, HSA had two negative CD bands 208 and $218 \mathrm{~nm}$, corresponding to the $\alpha$-helical structure in protein. ${ }^{23}$ While after HSA was mixed with a dispersion of GSH-Au NCs at $\mathrm{pH}$ 4.0, the bands at 208 and $218 \mathrm{~nm}$ almost disappeared, while a positive band emerged at $200 \mathrm{~nm}$ and a negative band appeared around $230 \mathrm{~nm}$, highlighting the substantial change in the secondary structure of the protein, which was caused by the interaction of HSA with the NCs. Contents of the secondary structures calculated from the CD spectrum of HSA were $50 \%$ for $\alpha$-helix, $12 \%$ for $\beta$-sheet, $13 \%$ for $\beta$-turn, and $25 \%$ for random coil. After being mixed with the NC dispersion, the content of $\alpha$-helix decreased dramatically to $9 \%$, while those of $\beta$-sheet, $\beta$-turn, and random coil increased to 37,25 , and $29 \%$, respectively. Such changes in secondary structure suggested that the Au NCs possibly bound HSA through hydrophobic interactions. ${ }^{27,28}$ The hydrophobic interaction of HSA with GSH-Au NCs was further illustrated by a control experiment, in which the enhanced $\mathrm{Au} \mathrm{NC}$ emission induced by HSA was greatly suppressed in the presence of guanidine hydrochloride $(\mathrm{GdnHCl})$, a denaturation agent that destroys the hydrophobic interaction between proteins and organic probes (Figure S3). ${ }^{22}$ Accordingly, the specific HSA-induced AEE effect of the Au NCs was possibly ascribable to the greater hydrophobicity of HSA compared to most of the other proteins. ${ }^{29}$

Time-resolved luminescence and X-ray photoelectron spectra (XPS) were acquired in order to shed light on the AEE effect. Decay curve of the Au NCs was fitted by a triexponential function, with lifetimes of $0.35 \mu \mathrm{s}$ (18.03\%), $1.14 \mu \mathrm{s}(47.85 \%)$, and $4.68 \mu \mathrm{s}$ (34.12\%) (Figure 3C). Such microsecond-level lifetimes indicated that emission of GSH$\mathrm{Au} \mathrm{NC}$ came from ligand-to-metal charge transfer/ligand-tometal-metal charge transfer, which resulted in radiative relaxation. $^{20,30}$ The lifetimes of the Au NCs were extended to $0.47 \mu \mathrm{s}$ (13.14\%), $2.17 \mu \mathrm{s}(46.15 \%)$, and $7.18 \mu \mathrm{s}(40.71 \%)$ in the presence of HSA. Such prolonged decay times indicated the suppressed ligand-related nonradiative relaxation in the excited state, which might contribute to the strikingly enhanced emission of the NCs induced by HSA. ${ }^{18,20}$ Moreover, $\mathrm{Au} 4 \mathrm{f}_{5 / 2}$ and $4 \mathrm{f}_{7 / 2}$ XPS peaks of the $\mathrm{Au}$ NCs centered at 88.1 and $84.3 \mathrm{eV}$ (Figure 3D), which indicated the presence of $\mathrm{Au}(0)$ and $\mathrm{Au}(\mathrm{I})$ in the NCs. Upon the addition of HSA, these peaks shifted to 83.5 and $86.9 \mathrm{eV}$, suggesting transformation of a part of the $\mathrm{Au}(\mathrm{I})$ into $\mathrm{Au}(0)$, which might also contribute to the AEE effect. ${ }^{31}$

\subsection{Method for HSA Determination Based on the} AEE Effect. Based on the HSA-induced AEE of the Au NCs, a luminescence method for quantitative determination of HSA was developed. Figure 4A reveals that emission intensities of the NCs increased with the increasing HSA concentration. Figure $4 \mathrm{~B}$ shows that the emission enhancement factor, $I / I_{0}$, is almost linear with the HSA concentration from 10 to $500 \mathrm{nM}$. The detection limit was $\sim 1.6 \mathrm{nM}$, estimated by the IUPAC method, which was comparable to those of organic probebased luminescence methods used for detecting HSA (Table $\mathrm{S} 1) \cdot{ }^{21-25}$ However, this present method is more facile and
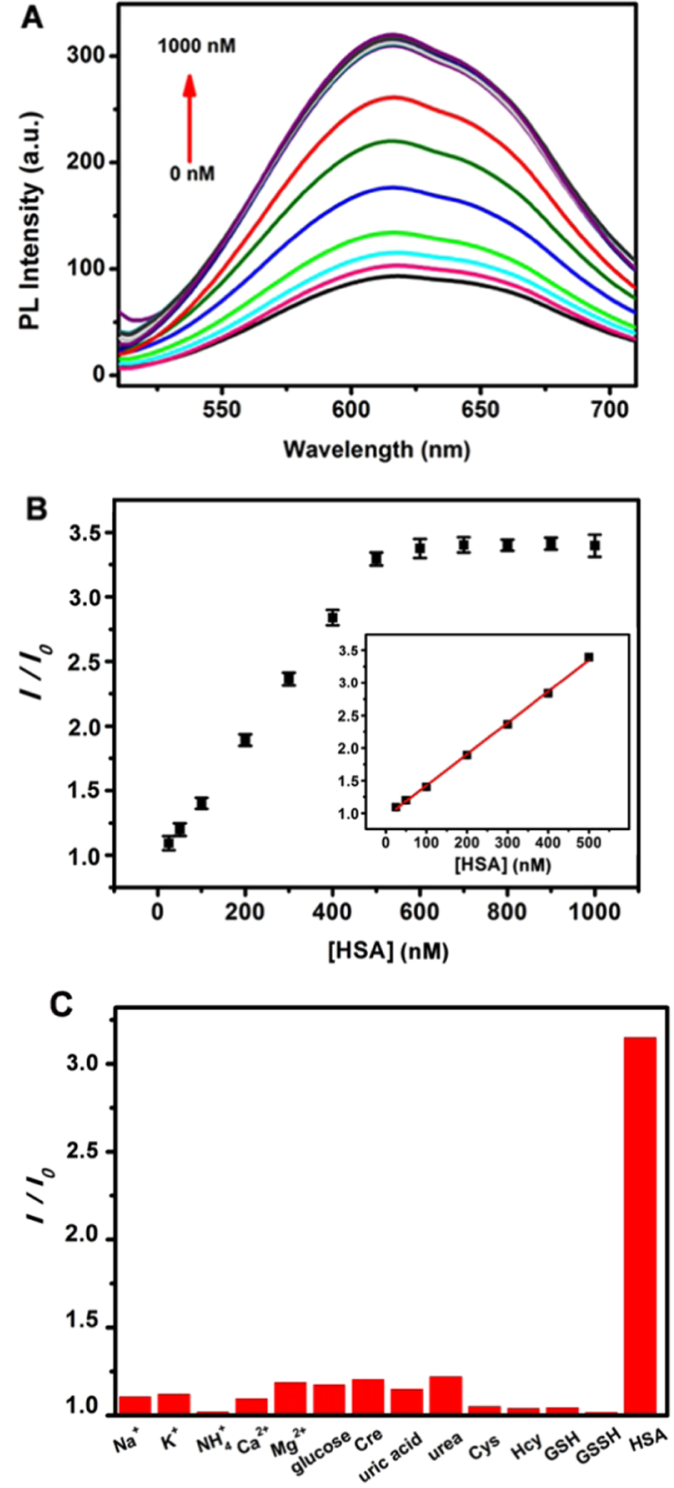

Figure 4. (A) Luminescence emission spectra and (B) enhancement factor, $I / I_{0}$, of GSH-Au NCs upon the addition of HSA $(0-1000$ $\mathrm{nM})$. Inset provides the linear calibration plot for HSA detection $(y=$ $0.0045 x-0.01, R^{2}=0.99$ ). (C) Histogram of $I / I_{0}$ upon the addition of $\mathrm{Na}^{+}$ions $(30 \mathrm{mM}), \mathrm{K}^{+}$ions $(30 \mathrm{mM}), \mathrm{NH}_{4}{ }^{+}$ions $(30 \mathrm{mM}), \mathrm{Ca}^{2+}$ ions $(3.0 \mathrm{mM}), \mathrm{Mg}^{2+}$ ions $(3.0 \mathrm{mM})$, glucose $(25 \mathrm{mM})$, creatinine (Cre, $0.5 \mathrm{mM})$, uric acid $(20 \mathrm{mM})$, urea $(0.2 \mathrm{M})$, cysteine (Cys, 500 $\mathrm{nM})$, homocysteine (Hcy, $500 \mathrm{nM})$, glutathione (GSH, $500 \mathrm{nM})$, oxidized glutathione (GSSH), and HSA (500 nM).

environment-friendly since no toxic organic solvents are required in the operations either to prepare the NCs or to detect the protein.

The influence of a variety of biologically relevant substances, including metal ions and bioactive small molecules, on emission intensities of the nanoclusters was then evaluated. Figure $4 \mathrm{C}$ reveals that the influence of these substances on emission intensities of the NCs was almost negligible. Consequently, this method should be capable of sensing HSA in practical samples, such as artificial urine. In a typical experiment, $100 \mu \mathrm{L}$ of artificial urine was spiked with defined concentrations of HSA, and then the mixtures were analyzed by using the AEE-based assay as well as a classical bicinchoninic acid (BCA) method. The results obtained 

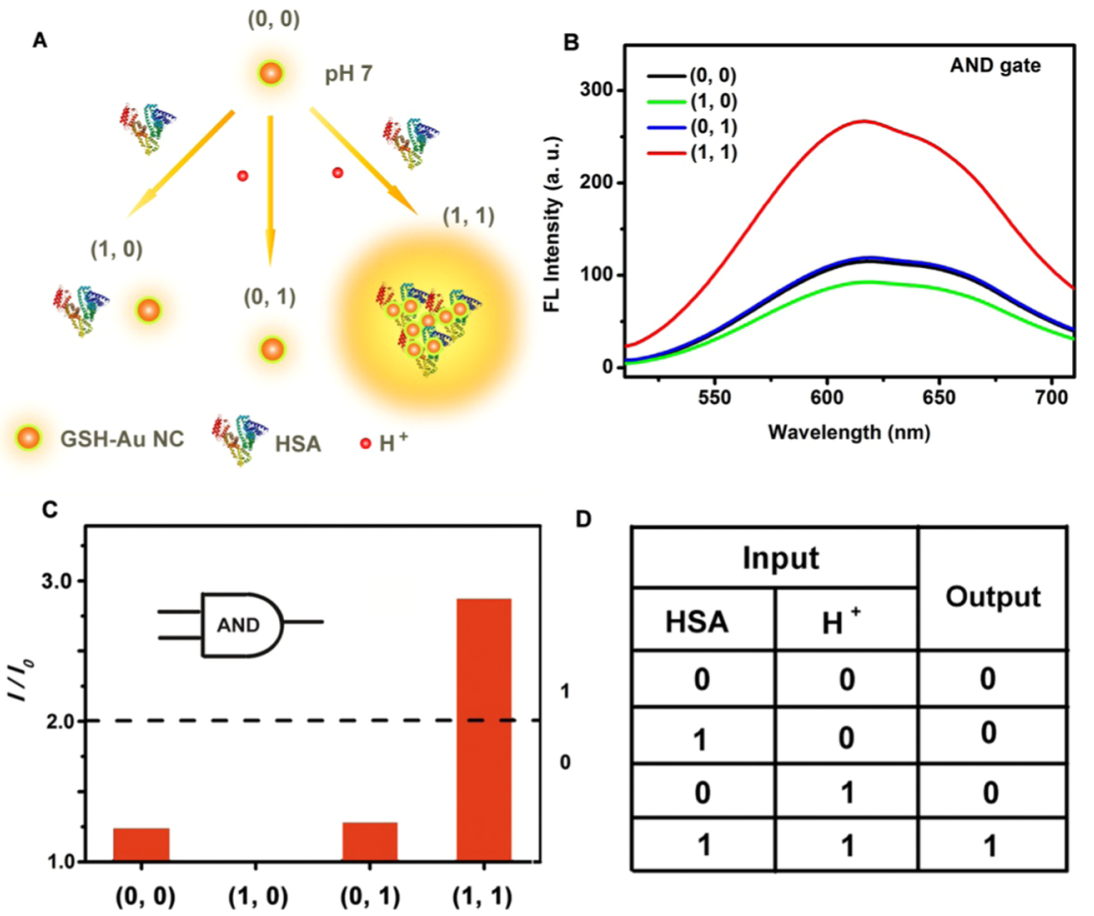

Figure 5. (A) Schematic representation for fabrication of the AEE-based AND logic gate. (B) Luminescence emission spectra, (C) $I / I_{0}$, and (D) truth table for the AEE-based AND logic gate.
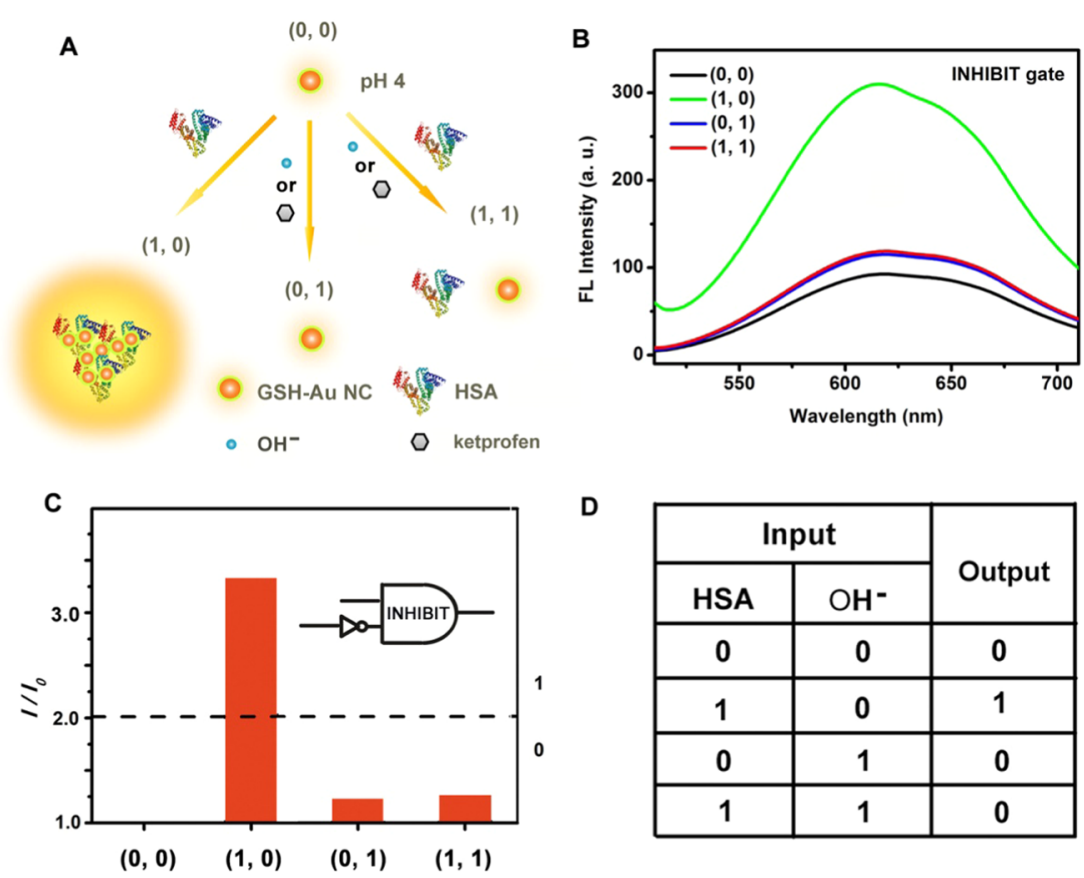

D

\begin{tabular}{|c|c|c|}
\hline \multicolumn{2}{|c|}{ Input } & \multirow{2}{*}{ Output } \\
\cline { 1 - 2 } HSA & $\mathrm{OH}^{-}$ & \\
\hline 0 & 0 & 0 \\
\hline 1 & 0 & 1 \\
\hline 0 & 1 & 0 \\
\hline 1 & 1 & 0 \\
\hline
\end{tabular}

Figure 6. (A) Schematic representation for fabrication of the AEE-based INHIBIT logic gate. (B) Luminescence emission spectra, (C) $I / I_{0}$, and (D) truth table for the AEE-based INHIBIT logic gate.

from the concentration calibration curve (Figure S4) for the AEE-based assay were consistent with those acquired using the BCA method (Table S2). In addition, our method exhibited excellent recoveries (96.0-98.7\%) and relative standard deviations $(<5.0 \%)$, highlighting its potential use for HSA detection in real samples.

2.4. AEE-Based Design of Molecular Logic Gates. Logic gates that transform chemical/biological input into measurable output have attracted significant attention due to potential applications to information processing at the molecular level. ${ }^{32,33}$ Based on the AEE effect associated with the Au NCs induced by HSA, we designed a platform capable of performing molecular logic operations. To implement the logic function, AEE-related factors such as $\mathrm{HSA}$ and $\mathrm{pH}\left(\mathrm{H}^{+}\right.$, $\mathrm{OH}^{-}$) were employed as inputs. The absence and presence of the input were designated to be " 0 " and " 1 ", respectively. On the other hand, the weak luminesce associated with dispersed GSH-Au NCs was defined to be output 0 , while the enhanced 
emission associated with the $\mathrm{Au}$ NC-HSA aggregates was defined to be output 1 , with a threshold $I / I_{0}$ value of 2.0. As shown in Figure 5, an AND gate that provides an output of 1 when both inputs are 1 was fabricated using the $\mathrm{pH}$-dependent AEE effect. The Au NC dispersion at $\mathrm{pH} 7.0$ was defined to be the initial state. The two inputs for the AND gate were set to be HSA and $\mathrm{H}^{+}$. The Au NCs exhibited poor luminescence without any input (output 0 ). The addition of a single input, either HSA or $\mathrm{H}^{+}$ions, into the $\mathrm{Au} \mathrm{NC}$ dispersion ( $\mathrm{pH}$ 7.0) was unable to trigger the AEE effect (output 0). Only when both inputs, that is, HSA and $\mathrm{H}^{+}$, were added simultaneously was AEE observed (output 1), which supported an AND function that transforms biochemical information, that is, the presence of HSA and environmental $\mathrm{pH}$ change, into a detectable luminescence signal.

An INHIBIT gate, in which the output is 1 when only one input is 1 (and the other input is 0 ), was constructed next. As shown in Figure 6, the initial state of the INHIBIT gate was set to be the GSH-Au NC dispersion at $\mathrm{pH}$ 4.0. HSA was used as one input and $\mathrm{OH}^{-}$as the other. The GSH-Au NCs exhibited AEE with only HSA as the input, to give an output signal of 1 . The addition of $\mathrm{OH}^{-}$alone resulted in negligible change of the weak luminesces of the GSH-Au NCs (output 0). However, when the gate was subjected to both inputs together, AEE was inhibited by the neutral $\mathrm{pH}$ condition caused by the addition of $\mathrm{OH}^{-}$(output 0 ); hence, this configuration corresponded to an INHIBIT logic gate. The $\mathrm{OH}^{-}$ions can be replaced by another input capable of suppressing the AEE effect when designing INHIBIT gates. For example, an AEE-based INHIBIT gate can also be designed using ketoprofen instead of $\mathrm{OH}^{-}$ions as an input. Ketoprofen is a nonsteroidal antiinflammatory drug that can competitively bind to HSA, ${ }^{27,34}$ thereby preventing interactions between HSA and the NCs (Figures 6A and S5). Considering that HSA is a model protein in molecular biology, the AEE-based INHIBIT gate might be potentially useful for the screening of drugs and the evaluation of their physiological activities.

\section{CONCLUSIONS}

In summary, we demonstrate that HSA is effective to induce the aggregation-enhanced emission (AEE) of GSH-Au NCs. To the best of our knowledge, this is the first study of a protein-triggered AEE effect of the Au NCs. It is identified that both hydrophobic and electrostatic interactions between HSA and the Au NCs contribute to the AEE effect. Such an AEE effect involving the Au NCs is suitable for the detection of HSA. Moreover, molecular platforms capable of performing AND and INHIBIT logic operations are fabricated based on the AEE effect. We expect that this study will provide new insight into protein sensing and offers an experimental basis for the future development of logic devices for molecular information processing and biomedical research.

\section{EXPERIMENTAL SECTION}

4.1. Materials and Instruments. Hydrogen tetrachloroaurate(III) ( $\geq 99.9 \%)$, HSA, BSA, ovalbumin, lipase and guanidine hydrochloride were purchased from Sigma. GSH, hemoglobin, horseradish peroxidase, lysozyme, RNase A, carbonic anhydrase, immunoglobulin G, trypsin, and BCA kit were purchased from Sangon Biotech. Phosphate buffers (20 $\mathrm{mM}$ ) with $\mathrm{pH} 4.0,5.0,6.0,7.0,8.0$, and 9.0 were prepared by mixing solutions of $\mathrm{Na}_{2} \mathrm{HPO}_{4}(20 \mathrm{mM})$ and $\mathrm{NaH}_{2} \mathrm{PO}_{4}(20$ $\mathrm{mM})$. The buffer $\mathrm{pH}$ was tuned by $\mathrm{HCl} / \mathrm{NaOH}$ solution.

Luminescence study was performed on a Shimadzu RF5301PC fluorometer. Time-resolved luminescence spectra were measured on an Edinburgh FS 920 fluorometer. UVvis spectra measurement was performed on a Shimadzu UV1800 spectrophotometer. TEM micrographs were obtained by a FEI Tecnai G2-Twin microscope. XPS were obtained using an ESCALAB-MKII spectrometer. CD spectra were carried out on a Bio-Logic MOS 500 circular dichroism spectrometer. Secondary structural contents were calculated by using the Dicro 2000 program. DLS and $\zeta$-potential measurement were performed on a Brookhaven ZetaPlus apparatus.

4.2. Interactions of GSH-Au NCs with HSA. GSH-Au NCs were prepared following Xie's method. ${ }^{20}$ Typically, 2.0 $\mathrm{mL}$ of a $\mathrm{HAuCl}_{4}$ solution $(20 \mathrm{mM})$ was introduced into a solution of GSH $(3.3 \mathrm{mM}, 18 \mathrm{~mL})$, followed by heating to 70 ${ }^{\circ} \mathrm{C}$ for $24 \mathrm{~h}$. Next, $40 \mu \mathrm{L}$ of the NCs and $100 \mu \mathrm{L}$ of an aqueous solution of HSA at the required concentration were added to $860 \mu \mathrm{L}$ of phosphate buffer, followed by incubation for $15 \mathrm{~min}$ at ambient temperature prior to any experiment.

4.3. Molecular Logic Operations. 4.3.1. AND Gate. $\mathrm{GSH}-\mathrm{Au} \mathrm{NCs}(40 \mu \mathrm{L})$ were introduced to $860 \mu \mathrm{L}$ of phosphate buffer ( $\mathrm{pH}$ 7.0). The AND gate was implemented upon the introduction of different input combinations into the GSH-Au NC dispersion. (0, 0): $\mathrm{H}_{2} \mathrm{O}$; $(1,0): 500$ nM HSA; $(0,1): \mathrm{H}^{+}$, by addition of $0.1 \mathrm{mM} \mathrm{HCl}$ to $\mathrm{pH} 4$ and $(1,1): 500$ nM HSA and $\mathrm{H}^{+}$ions.

4.3.2. INHIBIT Gate. GSH-Au NCs $(40 \mu \mathrm{L})$ were introduced to $860 \mu \mathrm{L}$ of phosphate buffer ( $\mathrm{pH} 4.0)$. The INHIBIT gate was implemented upon the addition of different input combinations into the GSH-Au NC dispersion. (0, 0): $\mathrm{H}_{2} \mathrm{O} ;(1,0): 500 \mathrm{nM}$ HSA; $(0,1): \mathrm{OH}^{-}$ions, by addition of $0.1 \mathrm{mM} \mathrm{NaOH}$ to $\mathrm{pH} 7$ and $(1,1): 500 \mathrm{nM}$ HSA and $\mathrm{OH}^{-}$ ions.

\section{ASSOCIATED CONTENT}

\section{S Supporting Information}

The Supporting Information is available free of charge on the ACS Publications website at DOI: 10.1021/acsomega.8b01875.

UV-vis spectrum, luminescence emission spectrum of $\mathrm{GSH}-\mathrm{Au}$ NCs (Figure S1); optical images of the $\mathrm{Au}$ NC dispersion before and after the addition of HSA (Figure S2); luminescence emission spectra of GSH-Au $\mathrm{NCs}$ with and without $\mathrm{HSA} / \mathrm{GdnHCl}$ (Figure S3); concentration calibration curve in artificial urine (Figure S4); fabrication of INHIBIT logic gate by using ketoprofen (Figure S5); comparison of luminescent methods for serum albumin determination (Table S1); determination of HSA in artificial urine (Table S2) (PDF)

\section{AUTHOR INFORMATION}

\section{Corresponding Author}

*E-mail: wsyang@jlu.edu.cn.

ORCID $\odot$

Wensheng Yang: 0000-0003-2674-6751

Notes

The authors declare no competing financial interest. 


\section{ACKNOWLEDGMENTS}

We are grateful to Prof. Dayang Wang for valuable discussion and English revision. This work was supported by the National Natural Science Foundation of China (Nos. 21303071 and 51372097).

\section{REFERENCES}

(1) Shang, L.; Nienhaus, G. U. Gold nanoclusters as novel optical probes for in vitro and in vivo fluorescence imaging. Biophys. Rev. 2012, 4, 313-322.

(2) Nie, L.; Xiao, X.; Yang, H. Preparation and biomedical applications of gold nanocluster. J. Nanosci. Nanotechnol. 2016, 16, 8164-8175.

(3) Tao, Y.; Li, M.; Ren, J.; Qu, X. Metal nanoclusters: novel probes for diagnostic and therapeutic applications. Chem. Soc. Rev. 2015, 44, $8636-8663$.

(4) Xie, J.; Zheng, Y.; Ying, J. Y. Protein-directed synthesis of highly fluorescent gold nanoclusters. J. Am. Chem. Soc. 2009, 131, 888-889.

(5) Zhang, X.-D.; Luo, Z.; Chen, J.; Shen, X.; Song, S.; Sun, Y.; Fan, S.; Fan, F.; Leong, D. T.; Xie, J. Ultrasmall $A_{10-12}(S G)_{10-12}$ nanomolecules for high tumor specificity and cancer radiotherapy. Adv. Mater. 2014, 26, 4565-4568.

(6) Liao, H.; Liu, G.; Liu, Y.; Li, R.; Fu, W.; Hu, L. Aggregationinduced accelerating peroxidase-like activity of gold nanoclusters and their applications for colorimetric $\mathrm{Pb}^{2+}$ detection. Chem. Commun. 2017, 53, 10160-10163.

(7) Ou, G.; Zhao, J.; Chen, P.; Xiong, C.; Dong, F.; Li, B.; Feng, X. Fabrication and application of noble metal nanoclusters as optical sensors for toxic metal ions. Anal. Bioanal. Chem. 2018, 410, 24852498.

(8) Guan, G.; Zhang, S.; Cai, Y.; Liu, S.; Bharathi, M. S.; Low, M.; Yu, Y.; Xie, J.; Zheng, Y.; Zhang, Y.; Han, M. Convenient purification of gold clusters by co-precipitation for improved sensing of hydrogen peroxide, mercury ions and pesticides. Chem. Commun. 2014, 50, 5703-5705.

(9) Hofmann, C. M.; Essner, J. B.; Baker, G. A.; Baker, S. N. Proteintemplated gold nanoclusters sequestered within sol-gel thin films for the selective and ratiometric luminescence recognition of $\mathrm{Hg}^{2+}$. Nanoscale 2014, 6, 5425-5431.

(10) Liu, Y.; Ai, K.; Cheng, X.; Huo, L.; Lu, L. Gold-nanoclusterbased fluorescent sensors for highly sensitive and selective detection of cyanide in water. Adv. Funct. Mater. 2010, 20, 951-956.

(11) Biswas, A.; Banerjee, S.; Gart, E. V.; Nagaraja, A. T.; McShane, M. J. Gold nanocluster containing polymeric microcapsules for intracellular ratiometric fluorescence biosensing. ACS Omega 2017, 2, 2499-2506.

(12) Roy, S.; Palui, G.; Banerjee, A. The as-prepared gold clusterbased fluorescent sensor for the selective detection of As-III ions in aqueous solution. Nanoscale 2012, 4, 2734-2740.

(13) Cui, M. L.; Liu, J.; Wang, X.; Lin, L.; Jiao, L.; Zhang, L.; Zheng, Z.; Lin, S. Selective determination of cysteine using BSA-stabilized gold nanoclusters with red emission. Analyst 2012, 137, 5346-5351.

(14) Gao, M.; Tang, B. Fluorescent sensors based on aggregationinduced emission: recent advances and perspectives. ACS Sens. 2017, $2,1382-1399$.

(15) Wang, H.; Liu, G. Advances in luminescent materials with aggregation-induced emission (AIE) properties for biomedical applications. J. Mater. Chem. B 2018, 6, 4029-4042.

(16) Ji, L.; Guo, Y.; Hong, S.; Wang, Z.; Wang, K.; Chen, X.; Zhang, J.; Hu, J.; Pei, R. Label-free detection of $\mathrm{Pb}^{2+}$ based on aggregationinduced emission enhancement of Au-nanoclusters. RSC Adv. 2015, 5, 36582-36586.

(17) Li, B.; Wang, X.; Shen, X.; Zhu, W.; Xu, L.; Zhou, X. Aggregation-induced emission from gold nanoclusters for use as a luminescence-enhanced nanosensor to detect trace amounts of silver ions. J. Colloid Interface Sci. 2016, 467, 90-96.

(18) Yahia-Ammar, A.; Sierra, D.; Mérola, F.; Hildebrandt, N.; Le Guével, X. Self-assembled gold nanoclusters for bright fluorescence imaging and enhanced drug delivery. ACS Nano 2016, 10, 25912599.

(19) Arques, S. Human serum albumin in cardiovascular diseases. Eur. J. Intern. Med. 2018, 52, 8-12.

(20) Luo, Z.; Yuan, X.; Yu, Y.; Zhang, Q.; Leong, D. T.; Lee, J. Y.; $\mathrm{Xie}, \mathrm{J}$. From aggregation-induced emission of $\mathrm{Au}(\mathrm{I})$-thiolate complexes to ultrabright $\mathrm{Au}(0) @ \mathrm{Au}(\mathrm{I})$-thiolate core-shell nanoclusters. J. Am. Chem. Soc. 2012, 134, 16662-16670.

(21) Xu, Y.; Zhang, M.; Li, B.; Wang, W.; Wang, B.; Yang, Y.; Zhu, $\mathrm{H}$. A fluorescence probe acted on site I binding for human serum albumin. Talanta 2018, 185, 568-572.

(22) Hong, Y.; Feng, C.; Yu, Y.; Liu, J.; Lam, J.; Luo, K.; Tang, B. Quantitation, visualization, and monitoring of conformational transitions of human serum albumin by a tetraphenylethene derivative with aggregation-induced emission characteristics. Anal. Chem. 2010, 82, 7035-7043.

(23) Fan, X.; He, Q.; Sun, S.; Li, H.; Pei, Y.; Xu, Y. Nanoparticles self-assembled from multiple interactions: a novel near-infrared fluorescent sensor for the detection of serum albumin in human sera and turn-on live-cell imaging. Chem. Commun. 2016, 52, 11781181.

(24) Gui, W.; Chen, X.; Ma, Q. A novel detection method of human serum albumin based on CuInZnS quantum dots- $\mathrm{Co}^{2+}$ sensing system. Anal. Bioanal. Chem. 2017, 409, 3871-3876.

(25) Sun, H.; Xiang, J.; Zhang, X.; Chen, H.; Yang, Q.; Li, Q.; Guan, A.; Shang, Q.; Tang, Y.; Xu, G. A colorimetric and fluorometric dualmodal supramolecular chemosensor and its application for HSA detection. Analyst 2014, 139, 581-584.

(26) Kalanur, S. S.; Seetharamappa, J.; Katrahalli, U. Voltammetric and spectroscopic investigations on the mechanism of interaction of buzepide methiodide with protein. Colloids Surf., B 2010, 75, 75-79.

(27) Jisha, V. S.; Arun, K. T.; Hariharan, M.; Ramaiah, D. Siteselective interactions: squaraine dye-serum albumin complexes with enhanced fluorescence and triplet yields. J. Phys. Chem. B 2010, 114, 5912-5919.

(28) Chakraborty, S.; Joshi, P.; Shanker, V.; Ansari, Z. A.; Singh, S. P.; Chakrabarti, P. Contrasting effect of gold nanoparticles and nanorods with different surface modifications on the structure and activity of bovine serum albumin. Langmuir 2011, 27, 7722-7731.

(29) Kato, A.; Matsuda, T.; Matsudomi, N.; Kobayashi, K. Determination of protein hydrophobicity using a sodium dodecyl sulfate binding method. J. Agric. Food Chem. 1984, 32, 284-288.

(30) Wang, C.; Wang, Y.; Xu, L.; Shi, X.; Li, X.; Xu, X.; Sun, H.; Yang, B.; Lin, Q. A galvanic replacement route to prepare strongly fluorescent and highly stable gold nanodots for cellular imaging. Small 2013, 9, 413-420.

(31) Zhang, X.; Wu, F. G.; Liu, P.; Gu, N.; Chen, Z. Enhanced fluorescence of gold nanoclusters composed of $\mathrm{HAuCl}_{4}$ and histidine by glutathione: glutathione detection and selective cancer cell imaging. Small 2014, 10, 5170-5177.

(32) Huang, Z.; Wang, H.; Yang, W. Gold nanoparticle-based facile detection of human serum albumin and its application as an INHIBIT logic gate. ACS Appl. Mater. Interfaces 2015, 7, 8990-8998.

(33) Misra, A.; Srivastava, P.; Shahid, M. Fluorescent probe mimicking multiple logic gates and a molecular keypad lock upon interaction with $\mathrm{Hg}^{2+}$ and bovine serum albumin. Analyst 2012, 137, $3470-3478$

(34) Tayyab, S.; Zaroog, M. S.; Feroz, S. R.; Mohamad, S. B.; Malek, S. N. A. Exploring the interaction between the antiallergic drug, tranilast and human serum albumin: insights from calorimetric, spectroscopic and modeling studies. Int. J. Pharm. 2015, 491, 352358. 